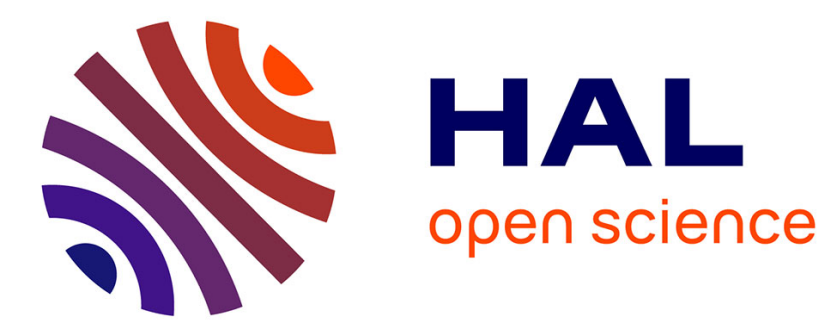

\title{
La pédagogie de l'inclusion dans le secteur de la petite enfance
}

\author{
Marie Andrys, Silvia Valentim
}

\section{To cite this version:}

Marie Andrys, Silvia Valentim. La pédagogie de l'inclusion dans le secteur de la petite enfance. Les Métiers de la petite enfance, 2018, 24, pp.14 - 15. 10.1016/j.melaen.2018.03.007 · hal-03487657

\section{HAL Id: hal-03487657 https://hal.science/hal-03487657}

Submitted on 20 Dec 2021

HAL is a multi-disciplinary open access archive for the deposit and dissemination of scientific research documents, whether they are published or not. The documents may come from teaching and research institutions in France or abroad, or from public or private research centers.
L'archive ouverte pluridisciplinaire HAL, est destinée au dépôt et à la diffusion de documents scientifiques de niveau recherche, publiés ou non, émanant des établissements d'enseignement et de recherche français ou étrangers, des laboratoires publics ou privés.

\section{다)(1) $(5$}

Distributed under a Creative Commons Attribution - NonCommerciall 4.0 International 
Dochead dossier

Sous-dochead L'inclusion, de l'intention à la pratique

Surtitre réflexion

\section{La pédagogie de l'inclusion dans le secteur de la petite enfance}

\section{Résumé}

La petite enfance est un secteur de l'action sociale en pleine mutation. Les politiques visent à accroître la capacité d'accueil des structures, alors que les publics accueillis sont très hétérogènes avec, parfois, des besoins d'accueil spécifiques. La notion de qualité est très présente dans ce secteur, sans que le sens qui lui est attribué soit toujours bien défini. Les répercussions des travaux de recherche et des pratiques autour de l'inclusion viennent interroger des logiques organisationnelles et professionnelles. Ce faisant, elles contribuent à placer l'accueil de la petite enfance au cœur d'un débat social majeur.

(C) 2018

Mots clés - diversité ; établissement d’accueil du jeune enfant, gestion ; inclusion ; pédagogie ; professionnel

\section{Auteurs}

\section{Marie Andrys a}

Cadre pédagogique, doctorante en sciences de l'éducation

Silvia Valentim ${ }^{\mathrm{a}, *, \mathrm{~b}}$

Directrice des études, chercheure associée

a Centre régional de formation des professionnels de l'enfance (CRFPE), 14 boulevard Vauban, 59042 Lille cedex, France

b Laboratoire EMA (École, Mutations, Apprentissages), EA 4507, Université de Cergy-Pontoise, ZAC des Barbanniers, avenue Marcel-Paul, 92230 Gennevilliers, France

*Auteur correspondant.

Adresse e-mail : silviavalentim@crfpe.fr (S. Valentim).

L'accueil des très jeunes enfants en France connaît des mutations importantes depuis les années 2000. Celles-ci vont dans le sens d'une volonté de développement du secteur, afin de répondre aux besoins des familles et atteindre les objectifs fixés par l'Europe [1]. Vient se joindre à cette visée quantitative du travail d'accompagnement de très jeunes enfants, l'ouverture du marché des crèches, la logique d'appel d'offre pour la délégation de service public (DSP) introduisant dans le secteur de nouveaux acteurs et organisations managériales. De nouvelles structures d'accueil pour les enfants de moins de 3 ans sont créées, comme les micro-crèches et les maisons d'assistantes maternelles (MAM), en même temps que s'opère une baisse dans les exigences de qualification professionnelle pour le secteur. Les critères réglementaires en termes de qualification par le diplôme s'assouplissent, il est désormais admis que seulement $40 \%$ du personnel de crèche soit en possession d'une qualification professionnelle reconnue [2]. 
À cela s'ajoute le fait qu'il n'est plus seulement question d'accueillir l'enfant et de lui apporter des soins que sa famille ne pourrait satisfaire, mais de se positionner en soutien aux familles, voire de participer à leur processus d'inclusion sociale.

\section{T1 Origines du concept d'inclusion}

Historiquement, le concept d'inclusion trouve ses racines dans l'accompagnement des personnes en situation de handicap, mais celui-ci peut faire référence à différentes réalités, qu'il soit physique, psychique, social ou culturel. Il en est de même pour l'inclusion dont la définition dépasse largement la question du handicap. En effet, dans le champ de la petite enfance, il est question d'inclure une grande diversité de jeunes enfants et de familles, dans le cadre d'un accueil d'accessibilité généralisée [2].

\section{T1 Contexte législatif et réglementaire}

TEG1 Sur le plan législatif, plusieurs évolutions des politiques publiques vont dans le sens de la lutte contre l'exclusion (ou le développement l'inclusion). En France, la loi d'orientation relative à la lutte contre l'exclusion, en 1998 [3], pose les jalons des décrets qui suivent : celui d'août 2000 [4] (décret d'application directe, dans le champ de la petite enfance, de la loi précitée) et ses différentes révisions, qui présentent l'accueil du jeune enfant comme une mission sociale (intégrer les enfants et parents exclus, lutter contre la pauvreté, lutter contre les inégalités sociales) et éducative (co-éduquer avec les parents, développer les compétences des enfants). TEG1 Par ailleurs, la fréquentation des lieux d'accueil collectifs, dès le plus jeune âge, est recommandée par l'OCDE [5] (Organisation de coopération et de développement économiques) comme moyen de lutte contre les inégalités sociales. Le rapport Giampino en 2016 [6] propose d'accélérer l'inclusion des enfants en situation de handicap, puisque cela reste l'un des défis majeurs pour le secteur.

TEG1 La loi $n^{\circ}$ 2005-102 pour l'égalité des droits et des chances, la participation et la citoyenneté des personnes handicapées définit, dans son article 114, la notion de handicap : "Constitue un handicap, au sens de la présente loi, toute limitation d'activité ou restriction de participation à la vie en société subie dans son environnement par une personne en raison d'une altération substantielle, durable ou définitive d'une ou plusieurs fonctions physiques, sensorielles, mentales, cognitives ou psychiques, d'un polyhandicap ou d'un trouble de santé invalidant. » [7]

TEG1 Le handicap est désormais défini comme " toute limitation d'activité ou restriction de participation à la vie en société » [8] subie, dans son environnement, par une personne, pour quelque raison que ce soit.

Si la définition de la loi de 2005 n'est pas exactement celle de la classification de l'Organisation mondiale de la santé (OMS) en 2001 [9] ${ }^{1}$, elle s'en inspire largement en reprenant trois dimensions essentielles :

- celle des fonctions physiques ou mentales et structures anatomiques, lesquelles se situent au plus près des organes ;

- celle des activités, dont la limitation désigne les difficultés de réalisation des actes concrets, plus ou moins complexes ;

- celle de la participation sociale, dont les restrictions entravent l'implication d'une personne dans la société. 
TEG1 Plus largement, l'un des principaux défis à relever pour l'Europe est de développer, dans tous les pays membres, des systèmes d'éducation "inclusifs". "La déclaration de Salamanque et la convention des nations unies sur les droits des enfants en [...] donnent une autorité internationale claire à la question de l'inclusion comme l'un des droits de l'homme " [10].

\section{T1 Conséquences pour les professionnels}

TEG1 Ces évolutions législatives et cette recommandation de l'OCDE mettent en tension les professionnels car ils sont confrontés à des réalités qui se complexifient, tout en disposant de moyens de plus en plus réduits pour y faire face, avec notamment la montée de la précarité [11], voire leur propre précariat [12]. De même, ces évolutions ont modifié des identités professionnelles qui n'étaient pas stabilisées [13]. Les professionnels de la petite enfance doivent quitter leur place "traditionnelle" pour aller au-devant des parents. II s'agit d'inventer ensemble, parents et professionnels, un nouveau chemin, un "entre-deux", ou encore un " espace de négociations " [14]. Les professionnels vivent une situation de crise : "les professionnels, tant du soin que dans le domaine du social ou de l'école, doivent ainsi élaborer leurs propres repères sur la base de leurs propres expériences » [15]. De plus, "les injonctions normatives des sociétés modernes, sommant aux individus de se conduire en sujets autonomes, fragilisent plus qu'elles ne renforcent les subjectivités contemporaines » [16]. Ainsi, le secteur de la petite enfance, sommé d'inclure la diversité, se fragilise.

TEG1 En effet, les évolutions en termes de gestion, prônant d'une certaine manière la standardisation des pratiques, contribue à cette fragilisation. Le secteur de la petite enfance se voit contraint de faire face à des logiques de rentabilité et d'efficience tout en assurant un accueil éducatif de "qualité", faisant preuve d'éthique professionnelle et d'ouverture à la diversité. Comment concilier des logiques paradoxales?

\section{T1 Une démarche porteuse d'espoir}

L'accueil de la diversité suscite des émotions contrastées où des mouvements affectifs forts, voire passionnés (peurs, enthousiasme, ambivalence), côtoient des sentiments d'incompétence ou d'inutilité. Les professionnels évoquent également le manque de temps et de moyens auxquels ils se sentent confrontés.

Aujourd'hui, en France, si l'inclusion n'est pas encore une réalité, des actions positives sont toutefois à l'œuvre. L'inclusion est-elle une utopie impossible dans un contexte professionnel qui se précarise ou, au contraire, y a-t-il une démarche professionnelle à mettre en valeur ? Une réflexion professionnelle est nécessaire, prenant en compte la complexité d'un accueil se voulant inclusif. Les travaux réalisés en ce sens en multi-accueil, en RAM, en médiathèque et en centre social, ainsi que des projets d'étudiants ${ }^{2}$ témoignent d'expériences riches et complexes, mais toutes sont porteuses d'espoirs et d'humanité.

Déclaration de liens d'intérêts

Les auteurs déclarent ne pas avoir de liens d'intérêts.

\section{Notes}

1 «Est handicapée toute personne dont l'intégrité physique ou mentale est passagèrement ou définitivement diminuée, soit congénitalement, soit sous l'effet de l'âge ou d'un accident, 
en sorte que son autonomie, son aptitude à fréquenter l'école ou à occuper un emploi s'en trouvent compromises ». Définition de 1980, revue en 2001.

2 comme ceux faisant suite au séminaire "Pédagogie de l'inclusion" qui se tient au CRFPE depuis 2010. Pour en savoir plus : http://www.crfpe.fr/formations/la-pedagogie-de-linclusion/

\section{Références}

[1] Collombet C. Accueil de la petite enfance en Europe : une progression modeste, des écarts entre États qui ne se résorbent pas. Étude de la Mission des relations européennes, internationales et de la coopération/ CNAF; 2015.

[2] Décret $n^{\circ} 2010-613$ du 7 juin 2010 relatif aux établissements et services d'accueil des enfants de moins de six ans.

https://www.legifrance.gouv.fr/eli/decret/2010/6/7/MTSA1014681D/jo/texte

[3] Loi no 98-657 du 29 juillet 1998 d'orientation relative à la lutte contre les exclusions. https://www.legifrance.gouv.fr/affichTexte.do?cidTexte=JORFTEXT000000206894

[4] Décret $n^{\circ} 2000-762$ du $1^{\text {er }}$ août 2000 relatif aux établissements et services d'accueil des enfants de moins de six ans et modifiant le code de la santé publique.

https://www.legifrance.gouv.fr/affichTexte.do?cidTexte=JORFTEXT000000218271\&categorieLie $\mathrm{n}=\mathrm{id}$

[5] OCDE. Résumé des principaux résultats de "Petite enfance, grands défis 2017 : indicateurs clés de l'OCDE sur l'éducation et l'accueil des jeunes enfants". 2017.

http://www.oecd.org/education/school/Resume-des-principaux-resultats-de-petite-enfancegrands-defis-2017.pdf

[6] Giampino S. Développement du jeune enfant, modes d'accueil, formation des

professionnels. Rapport. 2016. http://www.egalite-femmes-hommes.gouv.fr/wp-

content/uploads/2016/05/Rapport-Giampino-vf.pdf

[7] Loi $n^{\circ}$ 2005-102 du 11 février 2005 pour l'égalité des droits et des chances, la participation et la citoyenneté des personnes handicapées.

https://www.legifrance.gouv.fr/affichTexte.do?cidTexte=JORFTEXT000000809647\&categorieLie $\mathrm{n}=\mathrm{id}$

[8] Roussel P. Personnes en situations de handicap : concepts et définitions. La santé de I'homme. 2011;412:10-1.

[9] Organisation mondiale de la santé (OMS). Classification internationale des handicaps: déficiences, incapacités et désavantages (CIH). 1980, révisée en 2001.

http://apps.who.int/iris/handle/10665/41005?locale=fr

[10] Thomas G, Vaughan M. Inclusive education. Readings and reflections. Berkshire: Open University Press and New York: Two Pen Plaza; 2004. p128. In: Zay D. L'éducation inclusive. Une réponse à l'échec scolaire ? Paris: L'Harmattan; 2012.

[11] Valentim S. Entre gestion et éducation dans la petite enfance au Brésil et en France : des implications professionnelles sous tension. Thèse sous la direction de Gilles Monceau. Université de Cergy Pontoise. 2016.

[12] Castel R. Les ambiguïtés de l'intervention sociale face à la montée des incertitudes. Informations sociales. 2009/2;152:24-9.

[13] Moisset P. Écoute et dialogue autour des différences sociales et culturelles. Métiers de la petite enfance. 2016;230:7-9. 
[14] Cohen Emerique M. Pour une approche interculturelle en travail social. Théories et Pratiques. Rennes: Presses de l'EHESP;2011.

[15] Dubet F. Le déclin de l'institution. Paris: Seuil; 2002.

[16] Colloque. Travail, Santé, précarité, les transformations contemporaines des métiers des secteurs de la santé et du social à l'épreuve du sens. 16-17 mars 2017. https://tsp.univ-lille3.fr

\section{Illustration}

Andrys-Illus1.jpg

(C) Burger/Phanie

L'un des principaux défis à relever pour le secteur de la petite enfance est la mise en place de systèmes d'éducation "inclusifs". 


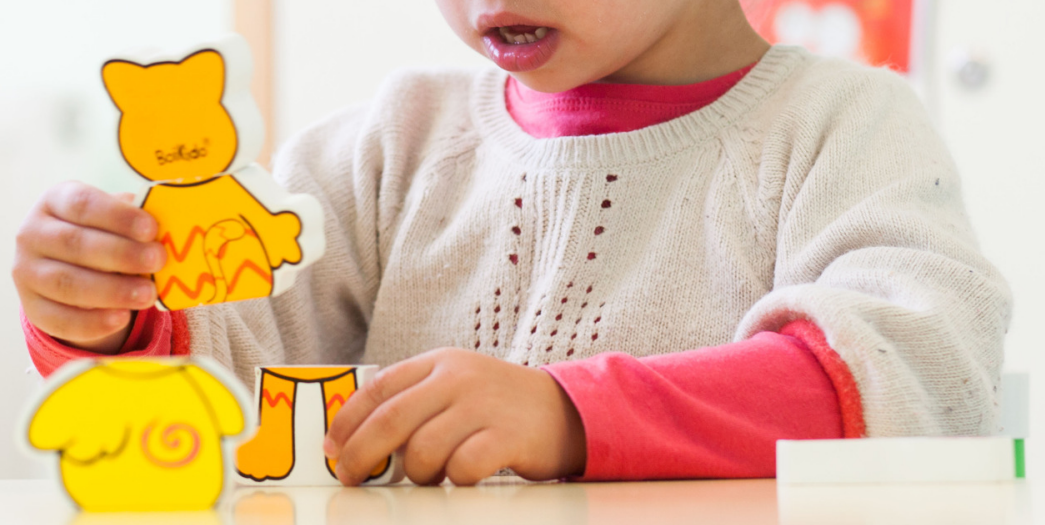

